\title{
El niño con Síndrome de Down en la Unidad de Cuidados Intensivos
}

\author{
Down Syndrome child in the Intensive care unit
}

\author{
Alejandro Donoso F. ${ }^{a}$, Soledad Montes F. ${ }^{b}$, Megan Neumann B. ${ }^{c}$, \\ Daniela Ulloa V.c, Dina Contreras E. ${ }^{c}$, Daniela Arriagada S. ${ }^{a}$
}

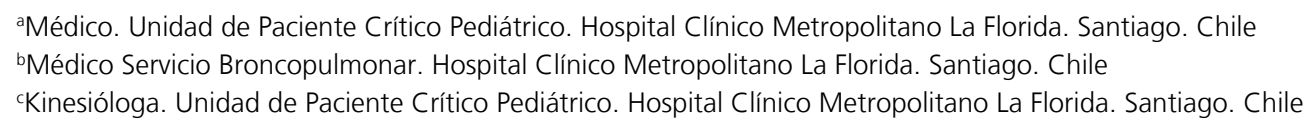

Recibido el 15 de marzo de 2017; aceptado el 31 de mayo de 2017

\section{Resumen}

El síndrome de Down es la alteración cromosómica más frecuente en los recién nacidos, con una alta incidencia en Chile. Esta condición presenta aspectos fisiológicos únicos, los cuales pueden afectar al niño durante su estadía en una Unidad de Cuidados Intensivos, posterior al período neonatal. En esta revisión abordamos aspectos actuales de la patología respiratoria, cardiovascular, infecciosa y neurológica, así como también consideraciones anestésicas y de analgesia postoperatoria, destete de la ventilación mecánica, inestabilidad columna cervical y pronóstico del niño críticamente enfermo portador de síndrome de Down. La evaluación de todas estas condiciones debe ser realizada cuando el paciente es ingresado a la Unidad de Cuidados Intensivos. El objetivo de la presente actualización es profundizar el conocimiento del diagnóstico y tratamiento de las potenciales complicaciones del niño con síndrome de Down durante su estadía en la unidad de paciente crítico.

\begin{abstract}
Down syndrome is the most common chromosomal abnormality in newborns, with a high incidence in Chile. This condition presents unique physiological aspects that should be known, which can affect the child during their stay in an Intensive Care Unit, beyond the neonatal period This review is focused on the respiratory, cardiovascular, infectious and neurological disorders. Anesthetic management and postoperative analgesia considerations, weaning from mechanical ventilation, cervical spine instability and prognosis of the critically ill child with Down syndrome are also analyzed. The evaluation of these conditions should be performed when the patient is admitted to the intensive care unit. The purpose of this update is to update the knowledge of the diagnosis and treatment of potential complications of children with Down syndrome during their stay in the unit of critical patient.
\end{abstract}

Palabras clave:

Síndrome de Down; trisomía 21; niño críticamente enfermo; cuidados intensivos

\section{Keywords:}

Down syndrome;

trisomy 21; critically ill children; intensive care 


\section{Introducción}

El síndrome de Down (SD) es la alteración cromosómica más frecuente en recién nacidos vivos. El Estudio Colaborativo Latinoamericano de Malformaciones Congénitas (ECLAMC) señaló una tasa de nacimientos de 1,88 por mil nacidos vivos ${ }^{1}$. En Chile, esta tasa es actualmente 2,47 por mil nacidos vivos, siendo la más alta de Sudamérica ${ }^{1,2}$.

En las últimas décadas los avances en la medicina infantil, el cambio de actitud hacia esta población, particularmente en aquellos niños con cardiopatía congénita sometidos a cardiocirugía y las políticas de salud pública con programas específicos de supervisión de salud han logrado un incremento notable en la sobrevida de los pacientes con $\mathrm{SD}^{3,4}$. Además, su ingreso a las unidades de cuidados críticos ocurre en forma más habitual.

Es conocido que el niño con SD presenta una mayor frecuencia de malformaciones congénitas cardíacas y gastrointestinales (atresia duodenal y páncreas anular), desórdenes orgánicos como enfermedad celíaca, hipotiroidismo, infecciones respiratorias y alteraciones hematológicas ${ }^{3}$. Este hecho se refleja en una mayor morbimortalidad y tasa de hospitalización que la población general ${ }^{5,6}$.

Diversos artículos han presentado resultados divergentes sobre la gravedad del niño con SD críticamente enfermo. A modo de ejemplo, en los pacientes con insuficiencia respiratoria que requieren de ventilación mecánica, los criterios para síndrome de distrés respiratorio agudo (SDRA) se presentan en forma más frecuente en niños con $\mathrm{SD}^{7}$. El riesgo de mortalidad por sepsis en estos pacientes aumenta un $30 \%$ tras corrección de factores de confusión ${ }^{8}$. Asimismo, las complicaciones respiratorias e infecciosas post-cardiocirugía son más comunes resultando en estadías más prolongadas en la unidad de cuidados intensivos (UCI) ${ }^{9}$.

Existen revisiones recientes del perfil de morbilidad del niño con SD en nuestro país ${ }^{5}$, pero no están enfatizadas en la morbilidad que ocasiona su condición de paciente críticamente enfermo. El objetivo de la presente comunicación es dar a conocer las particularidades fisiopatológicas, condiciones específicas y posibles patologías a encontrar en el niño con SD críticamente enfermo, para así capacitar al equipo de salud en su cuidado. Se hace exclusión del período neonatal y en lo referente a cuidados perioperatorios cardioquirúrgicos.

Se revisaron los artículos más relevantes a opinión de los autores en las bases de datos MEDLINE y Scielo. No se efectuó una revisión sistemática sobre el tema.

\section{Estrés oxidativo}

El estrés oxidativo es una parte fundamental de la biología del niño con SD. Entre los más de 500 genes del cromosoma 21, existe información funcional para alrededor de 150 de ellos. El cromosoma 21 contiene varios genes que han sido implicados en el estrés oxidativo incluyendo la superóxido dismutasa-1(SOD-1), factor de transcripción ETS-2, inhibidor de la calcineurina (región crítica del síndrome de Down-DSCR1), entre otros. Se ha señalado que los pacientes con SD presentan importantes diferencias en la susceptibilidad al estrés oxidativo ${ }^{10,11}$, describiendo una condición pro-oxidante sistémica ${ }^{12,13}$.

La mayoría de los pacientes con SD tienen una trisomía completa del cromosoma 21, existiendo una sobreexpresión del gen SOD1 (21qf22.1) el cual codifica para la forma soluble de la superóxido dismutasa1(SOD-1), una molécula clave en la anti-oxidación ${ }^{14}$. La relación entre SOD-1 y catalasa (CAT) más glutatión peroxidasa $(\mathrm{GPx})[\mathrm{SOD}-1 /(\mathrm{CAT}+\mathrm{GPx})]$ está aumentado en el SD, lo que favorece la generación en exceso de radicales libres de oxígeno (radical hidroxilo, $\mathrm{OH}$,) que ocasiona daño a las proteínas de la cadena electrónica en la membrana mitocondrial interna (menor eficiencia del metabolismo oxidativo), peroxidación de lípidos de membrana (daño celular) y daño del ADN mitocondrial ${ }^{10,11}$ (figura 1). En consecuencia, las células trisómicas son más sensibles al estrés oxidativo.

\section{Patología respiratoria}

El niño con SD puede presentar una gran variedad de patología respiratoria ${ }^{15}$. De esta manera los pacientes con SD son más susceptibles a infecciones del tracto respiratorio inferior, principalmente neumonía, las que motivan su hospitalización en $\mathrm{UCI}^{5,16}$. Esto es dado en parte a las particularidades en la anatomía de la vía aérea ${ }^{17}$, problemas derivados de la higiene oral ${ }^{18}$, hipotonía muscular, aspiración por trastorno de la deglución, y alteraciones en la inmunidad innata y adaptativa $^{19,20}$

Referente a la incidencia del SDRA en niños con SD se ha descrito que es mayor en comparación a la población pediátrica general ${ }^{7}$. Los mecanismos que lo explican no están del todo aclarados y pudiera deberse a una elevada tasa de apoptosis en estos niños. Al respecto, se ha sugerido que este incremento se relaciona con una menor capacidad para hacer frente al estrés oxidativo.

El estudio de Bertrand y cols. ${ }^{21}$, realizado en nuestro país, en una población de 24 pacientes con SD que presentaban síntomas respiratorios recurrentes y que fueron evaluados con fibrobroncoscopia, describió 


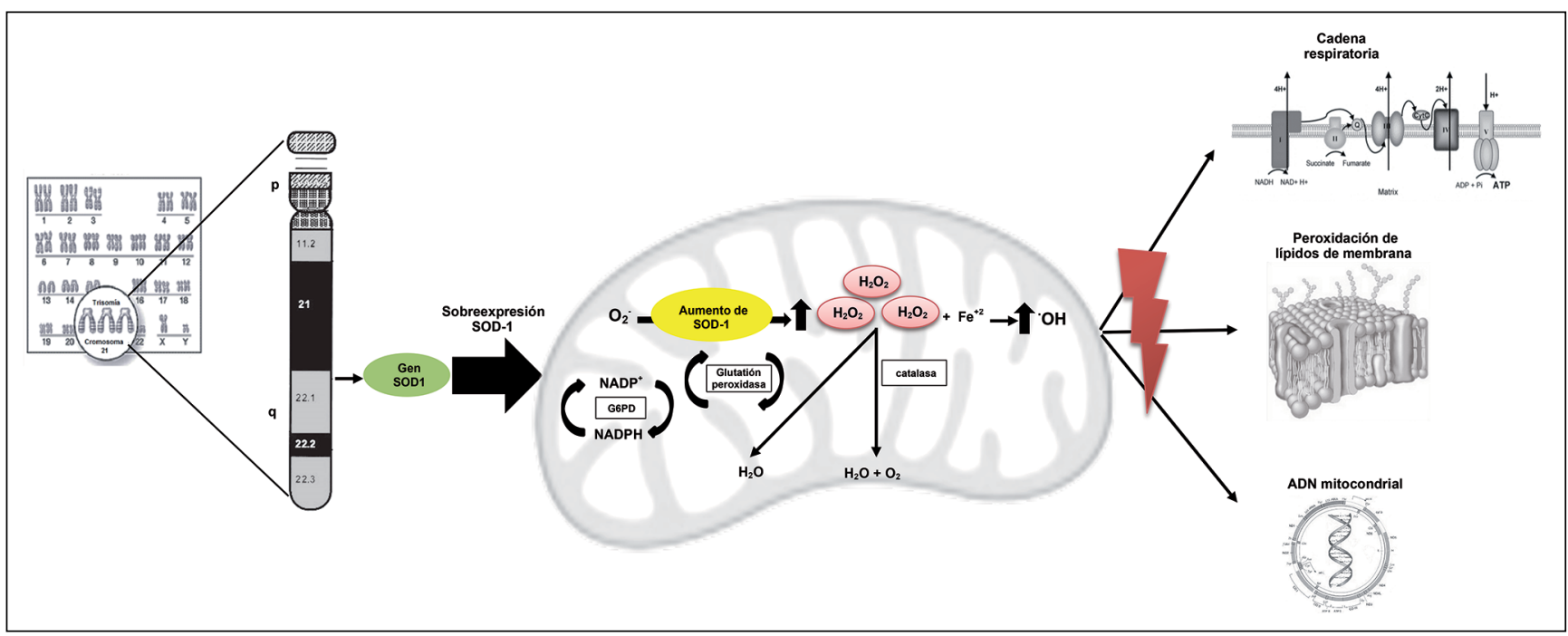

Figura 1. Representación esquemática del metabolismo anormal redox existente en los individuos con síndrome de Down. Las mitocondrias son la principal fuente de producción de aniones superóxidos $\left(\cdot \mathrm{O}_{2}{ }^{-}\right)$en la mayoría de las células. La mayoría de los pacientes con síndrome de Down presentan trisomía completa del cromosoma 21, donde 21q22.1 codifica para la forma soluble de la superóxido dismutasa 1 (SOD-1), molécula antioxidante clave. La relación [SOD-1/(CAT+GPx)] está aumentado en el síndrome de Down. Este desbalance crónico entre estas importantes enzimas antioxidantes y sus correspondientes sustratos favorece la generación en exceso de radicales hidroxilo, ( $\mathrm{OH})$. El estrés oxidativo se asocia con inhibición de la respiración mitocondrial, peroxidación lipídica y daño del ADN mitocondrial. SOD-1: superóxido dismutasa 1; CAT: catalasa; GPx: glutatión peroxidasa; $\mathrm{H}_{2} \mathrm{O}_{2}$ : peróxido de hidrógeno; $\mathrm{Fe}^{+2}$ : catión fierro ; • O2-: anión superóxido; $\mathrm{OH}$ : radical hidroxilo; $\mathrm{NADP}^{+}$: nicotinamida adenina dinucleótido fosfato; G6PD: glucosa-6-fosfato-deshidrogenasa.

que, dentro de las alteraciones en la vía aérea superior, la laringomalacia fue el hallazgo endoscópico más frecuente $(50 \%)^{21}$. Sin embargo, en una cohorte de 239 pacientes $^{22}$ el porcentaje fue menor $(6 \%)$. Por consiguiente, se debe considerar que estos pacientes presentan una vía aérea superior más lábil, y al usar benzodiacepinas u otros sedantes existe mayor riesgo de colapso de ésta. Esto se debe tener en cuenta al usar sedación en el paciente en ventilación no invasiva. También se observó que la presencia de traqueo y/o broncomalacia es la alteración de la vía aérea inferior más frecuente. Habitualmente es mayor a izquierda y debe sospecharse en caso de cardiopatía asociada (compresión bronquial). Su manifestación más frecuente es la atelectasia recurrente y fracaso de extubación.

En un subgrupo de pacientes sintomáticos respiratorio, se describió la existencia de quistes subpleurales (20-36\%) los cuales son múltiples, periféricos y en relación a cisuras, su tamaño varía de 1 a $4 \mathrm{~mm}$ de diámetro. Su manejo es conservador ${ }^{23}$.

Con respecto al desarrollo de hemorragia pulmonar, se puede presentar en forma aguda (hemoptisis o insuficiencia respiratoria) o subaguda (anemia ferropriva, hemosiderosis pulmonar idiopática, enfermedad intersticial pulmonar ${ }^{24}$. El mayor riesgo de hemorragia pulmonar se observa en el paciente con cardiopatía (cortocircuito de izquierda a derecha, disfunción mitral, obstrucción de vena pulmonar ${ }^{23}$.
Existen estudios que demuestran una mayor morbilidad por infecciones asociadas al virus respiratorio sincicial (VRS), observándose seis veces más hospitalizaciones durante los primeros dos años de vida, y en ausencia de otras patologías asociadas el riesgo es tres veces mayor. En relación a la gravedad, presentan dos veces más riesgo de necesidad de ventilación mecánica invasiva y mayor estadía hospitalaria. Esto se atribuye a la presencia de hipotonía muscular, mal manejo de secreciones, disfunción inmune, alteraciones en el desarrollo pulmonar y existencia de patologías asocia$\mathrm{das}^{25}$. Recientemente, se ha comunicado que estos niños presentan una mayor mortalidad para la infección grave por VRS ${ }^{26}$.

\section{Vía aérea e intubación endotraqueal}

El paciente con SD presenta microbraquicefalia y una vía aérea disminuida (vía aérea superior pequeña y macroglosia) por lo que la intubación puede ser dificultosa.

Con el incremento de la cirugía en estos niños se ha descrito en la literatura científica una asociación entre SD y desarrollo de estridor post-extubación. Dentro de las razones se encuentran: tamaño más pequeño y menor peso corporal, hipoplasia del tercio medio facial, estenosis subglótica congénita e hipotonía de la vía aérea ${ }^{22,27}$. 
La hipoplasia de tercio medio facial con una vía aérea nasal más pequeña y micrognatia, el desplazamiento medial de las amígdalas, la estrechez de nasofaringe y la hipotonía de la vía aérea, ubica a esta población en riesgo de presentar obstrucción de la vía aérea en diferentes niveles ${ }^{27}$.

El uso de máscara laríngea es recomendable en procedimientos cortos y de ser necesaria la intubación endotraqueal, se debe considerar la utilización de tubos endotraqueales (TET) más pequeños ${ }^{27}$. El estudio de Shott ${ }^{27}$ evaluó el tamaño apropiado del TET que debería utilizarse en estos niños. Sus resultados confirman que los niños con SD tienen una vía aérea más pequeña. Aparentemente se debe a una disminución global en el tamaño, no sólo de la vía aérea subglótica, sino también de la tráquea. En la tabla 1 se muestra las recomendaciones para los tamaños de los TET a utilizar.

\section{Patología de la vasculatura pulmonar}

La existencia de hipertensión pulmonar (HP) puede ser de naturaleza primaria ${ }^{28}$ o secundaria a la existencia de cardiopatía o hipoxia (apneas obstructivas del sueño, daño pulmonar crónico). Dentro de los mecanismos asociados, se atribuye un rol a la alteración del desarrollo de la vasculatura, polimorfismo genético que afectaría la producción de óxido nítrico y otros mediadores de la resistencia vascular pulmonar, entre otras causas c $^{23,29,30}$.

Se debe descartar la existencia de estenosis de las venas pulmonares, la que habitualmente origina una HP de pobre respuesta terapéutica. Su mecanismo causal sería la neo-proliferación de miofibroblastos. El diagnóstico se realiza por sondeo cardíaco, ya que en su mayoría no es evidente a la ecocardiografía. Su pronóstico es malo, con un alto riesgo de mortalidad (83\%) en caso de compromiso de 3 ó 4 venas pulmonares $^{31}$.

\section{Destete ventilatorio y fracaso de extubación}

Se debe tener presente que las anormalidades existentes en la vía aérea superior e inferior y los riesgos potenciales para el desarrollo de secuelas en la región subglótica derivadas del uso de un TET inadecuado pueden causar dificultades en el destete definitivo del ventilador y prolongar la estadía hospitalaria impactando negativamente el pronóstico vital ${ }^{32}$.

Se ha señalado que esta condición es un factor de riesgo por sí solo de fracaso de extubación (FE). En una importante casuística nacional de pacientes post operados cardíacos, un $7 \%$ de ellos presentaban $\mathrm{SD}$ con una tasa de FE de 22\% comparado con un $8,9 \%$ de los pacientes sin esta condición, alcanzando un valor sig-
Tabla 1. Recomendaciones para los tamaños de los tubos endotraqueales a utilizar en niños con síndrome de Down (Modificado de referencia 27)

\begin{tabular}{lc}
\hline Edad & Tamaño (DI, mm) \\
\hline Prematuro & $2,0-2,5$ \\
Término a 9 meses & $2,5-3,0$ \\
9 meses a 18 meses & $3,0-3,5$ \\
1,5 a 3 años & $3,5-4,0$ \\
4 a 5 años & $4,0-4,5$ \\
6 a 7 años & 5,0 \\
8-10 años & 5,5 \\
$10-11$ años & 5,5 \\
$12-13$ años & 6,0 \\
$\geq 14$ años & 6,5 \\
\hline
\end{tabular}

DI: diámetro interno; mm: milímetro.

nificativo como factor de riesgo para FE en los menores de seis meses de edad (OR: 5,7; 1,5-21,2) ${ }^{33}$.

Puede ser una opción a considerar en el niño con SD el uso de dexmedetomidina con el objetivo de minimizar o eliminar el uso de midazolam previo a la extubación.

\section{Patología cardíaca}

Las malformaciones más prevalentes asociadas a esta condición son las del sistema cardiovascular, con una incidencia que varía entre 42 a $48 \%$ para las malformaciones cardíacas ${ }^{34,35}$, siendo el hallazgo más frecuente el defecto aurículoventricular.

La presencia de cardiopatía congénita en el niño con SD es la principal causa de ingreso a UCI cardioquirúrgica. Esta temática se encuentra fuera del objetivo de esta actualización, no obstante, el lector puede encontrar revisiones sobre esta materia ${ }^{9,36,37}$.

Se ha descrito una mayor frecuencia de bradicardia en estos niños al recibir inducción anestésica con sevoflurano, independiente de la coexistencia de cardiopatía ${ }^{38}$. Habitualmente ésta es de fácil corrección y no se asocia a un peor pronóstico ${ }^{39}$.

Como se señaló previamente la incidencia de HP es mayor en niños con $\mathrm{SD}^{29,30}$, donde la alta prevalencia de cardiopatía congénita es un contribuidor significativo.

La progresiva incorporación del uso de ventilación no invasiva ha proporcionado una mejoría clínica significativa en los pacientes con falla cardiaca congestiva e hipertensión pulmonar y un probable impacto benéfico en la mortalidad ${ }^{40}$. Finalmente, se ha demostrado en importantes estudios de cohorte que el neonato y niño con SD presenta el mismo ${ }^{9}$ o menor riesgo de muerte a corto plazo $^{41}$ que el niño sin SD post cardiocirugía. 
Tabla 2. Factores inmunológicos y no inmunológicos que potencialmente aumentan la susceptibilidad a infecciones en el niño con síndrome de Down (Adaptada de referencias 19 y 20)

Factores inmunológicos

Factores no inmunológicos

Linfopenia

Alteraciones estructurales de la vía aérea

Respuesta específica de Ac a la inmunización reducida

Menor tamaño del timo Manejo inadecuado de secreciones bronquiales

Niveles de IgA total y específica en saliva disminuida Reflujo gastroesofágico

Niveles de subclase lgG4 total y específica en saliva disminuida

Defecto en la quimiotaxis de neutrófilos

Ac: anticuerpo; Ig: Inmunoglobulina.

\section{Patología infecciosa}

Los pacientes con SD presentan una elevada frecuencia de infecciones, particularmente del tracto respiratorio, las que se caracterizan por ser de duración prolongada y mayor gravedad ${ }^{42}$. Garrison y cols. ${ }^{8}$ comunicaron un $30 \%$ de incremento en el riesgo de mortalidad en la población infantil con SD comparado con niños sin SD. Esto se debe tanto a factores inmunológicos ${ }^{20} \operatorname{como~no~inmunológicos~}^{19}$ (tabla 2).

La homeostasis del zinc es crítica tanto para una adecuada inmunidad innata como para una función linfocitaria normal (inmunidad adaptativa) ${ }^{43}$, permitiendo postular que su alteración se puede relacionar con la disfunción inmune del niño séptico.

Se ha evidenciado en pacientes críticos que niveles plasmáticos bajos de zinc se correlaciona con la magnitud de la falla orgánica ${ }^{44}$. Por otra parte, ha sido demostrado en un modelo experimental animal y en pacientes sépticos que la presencia de menores niveles séricos de zinc se asocia a mayor mortalidad ${ }^{45}$.

Se ha postulado que los niveles de zinc en los niños con SD se encuentran disminuidos, lo que se demostró en un estudio con una pequeña casuística, que incluyó 35 pacientes con esta condición, entre 4 a 11 años de edad $^{46}$. El rol patobiológico del zinc en el niño con SD y shock séptico no está dilucidado.

Estudios de asociación del genoma completo (GWAS, del inglés Genome-Wide Association Study) han comunicado que el shock séptico en el niño se caracteriza por una precoz, persistente y concomitante represión de programas genéticos relacionados con el sistema inmune adaptativo y la biología del $z_{\text {inc }}{ }^{43,45}$, identificándose posteriormente que una subclase de esta población presentó un fenotipo clínico asociado a un peor pronóstico vital. Dado la deficiencia que presenta en la inmunidad adaptativa el paciente con SD, la cual se exacerba en el paciente con shock séptico, se debiera disponer a futuro mediante una subclasificación a nivel genómico, de una terapia específicamente orientada.

\section{Trastornos neurológicos}

Además de los hallazgos transversales en los niños con SD como son la hipotonía y el retraso cognitivo, existen otras complicaciones neurológicas a tener presentes. Según los trastornos neurológicos a evaluar se ha descrito una prevalencia variable. Schieve y cols. ${ }^{47}$ señalaron una frecuencia de trastornos neurológicos de $26 \%$ para niños entre 3 a 17 años y en una reciente casuística nacional de dos centros hospitalarios ${ }^{48}$ la cual incluyó a pacientes neonatales, se describió una prevalencia de $39 \%$, destacando los trastornos oculomotores $(26 \%)$ y la epilepsia (12\%), presentando esta última una frecuencia mayor que la reportada en otras comunicaciones $^{49}$. Gaete y cols. ${ }^{48}$ señalan que el tipo predominante de epilepsia fue la generalizada, y lo más frecuente en este grupo fue el espasmo infantil, hallazgo concordante con otras publicaciones ${ }^{50,51}$. Referente al Síndrome de West en el niño con SD se ha descrito una mejor respuesta a la terapia esteroidal que para la población general $^{51,52}$.

Recientemente un estudio de cohorte, comparó a pacientes hospitalizados niños y adultos, con SD y sin esta condición. La población estudiada corresponde a una muestra hospitalaria de 20.405 pacientes, de los cuales el 20\% del total corresponde a personas con SD. Este trabajo señaló que los pacientes con esta condición tienen asociado un alto riesgo de accidente cerebro vascular en todas las edades. En el caso de desarrollo de accidente cerebrovascular isquémico en pacientes con $\mathrm{SD}$, este aparece principalmente por el riesgo cardioembólico. El mayor riesgo de accidente cerebrovascular hemorrágico y menor riesgo de eventos coronarios (en hombres) con SD siguen sin explicación ${ }^{53}$. Sin embargo, en esta casuística, la población de pacientes con esta condición tiene una representación excesiva, por lo cual los resultados no deben extrapolarse a nivel poblacional.

Además se debe destacar la mayor frecuencia descrita para el síndrome de Moyamoya en niños con SD que en la población pediátrica general ${ }^{54}$. Este se pre- 
senta habitualmente con síntomas de carácter isquémico. La causa de esta asociación aún no es totalmente aclarada, atribuyéndose un rol a la displasia vascular, condiciones protrombóticas, fenómenos de autoinmunidad, síntesis de colágeno tipo VI y sobreexpresión de proteínas que regulan la fisiología arterial. Su tratamiento es la cirugía de revascularización cerebral $^{55}$.

\section{Inestabilidad de columna cervical}

Predecir el riesgo de inestabilidad cervical en un paciente puede ser dificultoso. Diversas comunicaciones señalan una frecuencia de inestabilidad atlantoaxial y atlantooccipital variable entre $15 \%$ a $30 \%{ }^{56}$.

En cuanto a la sintomatología para identificar a pacientes con inestabilidad atlantoaxial esta es muy variable y de escasa frecuencia $(1-2 \%)^{57}$. Esto implica que la historia clínica y el examen físico pueden no presentar utilidad para detectarla. La evaluación radiológica también es difícil en los niños menores de tres años de edad, dado que la columna está inadecuadamente osificada para permitir mediciones exactas, además de presentar modificaciones de la anatomía cervical a medida que el niño crece. El índice más empleado en su objetivación es el intervalo atlantoodontoideo ${ }^{56}$.

Los síntomas y signos de la inestabilidad atlantoaxial son usualmente crónicos, resultante de la compresión de la médula espinal y/o de las raíces nerviosas. Sin embargo, la compresión aguda del cordón cervical superior es un potencial escenario de riesgo ante hiperextensión cervical durante procedimientos como laringoscopia, broncoscopia o esofagoscopia ${ }^{17}$. De esta manera algunos especialistas prefieren la intubación fibroóptica o la estabilización en línea de la columna cervical en pacientes con riesgo. Además se debe efectuar un examen neurológico básico para asegurar simetría de movimientos y fuerza de las extremidades, el cual debe ser repetido en el paciente que puede haber sufrido daño cervical.

En la actualidad, la Academia Americana de Pediatría no recomienda el uso rutinario de radiografías de columna cervical para el niño asintomático. No obstante, habitualmente la decisión de disponer o no de una evaluación preoperatoria radiológica permanece a discreción del anestesiólogo o cirujano.

\section{Consideraciones anestésicas}

Además de la elevada prevalencia de cardiopatías congénitas y malformaciones gastrointestinales, las que habitualmente requieren cirugía durante los primeros días o semanas de vida, existen otras condiciones de resolución quirúrgica (malformaciones menores, procedimientos dentales, etc.) que se presentan frecuentemente, o también por la necesidad de efectuar procedimientos diagnósticos prolongados, el niño con SD debe ser sometido a anestesia o sedación profunda en forma más reiterada que otros individuos, siendo necesario maximizar las condiciones antes, durante y después de la cirugía ${ }^{58}$. De esta manera, generalmente estos niños necesitan ser ingresados a la UCI para su recuperación.

Se ha demostrado que la incidencia de complicaciones relacionadas con la anestesia general es significativamente mayor en estos pacientes para procedimientos no cardíacos, destacando la presencia de bradicardia grave, obstrucción de la vía aérea y estridor post instrumentalización ${ }^{59}$.

Aspectos fisiológicos únicos del niño con SD pueden potencialmente afectar la seguridad perioperatoria, por lo tanto, es necesario una adecuada comunicación entre anestesista e intensivista. Se debe colocar especial énfasis en la inestabilidad atlantoaxial ${ }^{60}$, hipertensión pulmonar, patología cardiovascular, obstrucción de la vía aérea superior, alteraciones hematológicas y antecedente de prematurez o bajo peso de nacimiento ${ }^{58}$.

Asimismo, presentan mayor riesgo postoperatorio de apneas, episodios hipoxémicos y edema pulmonar agudo post-obstructivo ${ }^{23}$. En la literatura científica, además se describe la coexistencia de SD con síndrome de hipoventilación central congénito, por lo cual es importante indagar tanto en la presencia de apneas centrales y obstructivas en la anamnesis.

Ante la necesidad de sedación para procedimientos en pacientes con apnea obstructiva del sueño, la combinación de ketamina-dexmedetomidina es una buena alternativa.

Finalmente, de ser posible se debe considerar la combinación de dos o más procedimientos quirúrgicos compatibles bajo un solo evento anestésico, disminuyendo así las potenciales complicaciones de la inducción anestésica, extubación y etapa postoperatoria.

\section{Analgesia postoperatoria}

Existen datos limitados y contradictorios, la mayoría de ellos provenientes de ensayos clínicos en pacientes sometidos a cirugía cardíaca, referentes a la analgesia necesaria en el niño con SD en comparación al que no presenta esta condición. No obstante, estudios recientes confirmaron dosis analgésicas comparables en pacientes neonatales y pediátricos, en lo referente a la dosis acumulativa de opioides a las 24 y $96 \mathrm{~h}^{61}$ y similares requerimientos de fármacos sedantes y analgésicos ${ }^{62,63}$.

Se debe tener presente la dificultad en el niño con 
SD para valorar adecuadamente el dolor y, por ende, su dependencia para una correcta evaluación y tratamiento.

\section{Pronóstico}

Uno de los factores claves que influyen en la mortalidad del paciente crítico es la presencia de diversas fallas orgánicas. En la actualidad, se reconoce a la disfunción multiorgánica como una respuesta adaptativa originada por la modificación de la respuesta inmune, neuroendocrina y estado redox sobre la función mitocondrial.

Esta respuesta adaptativa predice una compleja relación entre la gravedad de la disfunción orgánica y el resultado: aquellos pacientes capaces de desencadenarla precozmente tendrán una tendencia a evitar la muerte temprana en un episodio de enfermedad crítica, pero con un mayor requerimiento de soporte orgánico. La eventual sobrevivencia estará determinada por la capacidad de recuperación mitocondrial una vez que el evento inicial ha cesado.

Debe considerarse que los niños con SD presentan diferentes características en su inmunidad ${ }^{8}$, en la homeostasis redox ${ }^{10}$ y neuroendocrinas, lo que origina distintos umbrales para el inicio y resolución de la falla orgánica, durante el desarrollo de la enfermedad crítica.

Una de las principales revisiones sobre la evolución y pronóstico del niño con SD críticamente enfermo señaló una mayor necesidad de soporte cardiovascular, ventilatorio y renal que lo sugerido por la gravedad de la enfermedad al momento de la admisión, como también una mayor estadía en UCI y una tasa de mortalidad dependiente de la duración de ésta ${ }^{32}$.

Una reciente comunicación demostró que poseer esta condición no confiere mayor riesgo de mortalidad entre pacientes críticos neonatales y pediátricos menores de dos años de edad. No obstante, corroboró que estos pacientes presentan un riesgo aumentado de mortalidad en relación al incremento de su estadía hospitalaria $^{64}$.

Por otra parte, un estudio retrospectivo francés, señaló una mayor gravedad y elevada mortalidad que la predicha por el score PELOD (Paediatric logistic organ dysfunction) en niños con SD críticamente enfermos admitidos a UCI por razones médicas (no quirúrgicas), relacionada principalmente a falla respiratoria ${ }^{65}$.
Finalmente, aún falta información relacionada a la calidad de vida del niño luego de su alta de UCI.

\section{Conclusiones}

Las características únicas de su anatomía y patofisiología en el niño con SD plantean una serie de consideraciones y eventuales problemas que deben ser anticipados durante su estadía en UCI. Los niños con SD constituyen un importante grupo de pacientes, donde su admisión por causas médicas se asocia a morbimortalidad habitualmente incrementada por la presencia de enfermedades o condiciones respiratorias asociadas.

Estos pacientes presentan importantes diferencias en la susceptibilidad al estrés oxidativo, reconociendo una condición pro-oxidante sistémica. La incidencia de SDRA es mayor que la observada en la población pediátrica general y aún queda por definir el exacto rol patobiológico del zinc en el niño con SD y shock séptico.

Los niños con SD necesitan un mayor soporte orgánico que el predicho por su gravedad al momento de su admisión a UCI, a su vez que el riesgo de mortalidad no depende de poseer la condición de SD, pero sí de la duración de su estadía en ésta.

\section{Responsabilidades éticas}

Protección de personas y animales: Los autores declaran que los procedimientos seguidos se conformaron a las normas éticas del comité de experimentación humana responsable y de acuerdo con la Asociación Médica Mundial y la Declaración de Helsinki.

Confidencialidad de los datos: Los autores declaran que han seguido los protocolos de su centro de trabajo sobre la publicación de datos de pacientes.

Derecho a la privacidad y consentimiento informado: Los autores han obtenido el consentimiento informado de los pacientes y/o sujetos referidos en el artículo. Este documento obra en poder del autor de correspondencia.

\section{Conflicto de intereses}

Los autores declaran no tener conflicto de intereses. 


\section{Referencias}

1. Nazer J, Cifuentes L. Estudio epidemiológico global del síndrome de Down. Rev Chil Pediatr. 2011;82(2):10512.

2. Nazer J, Antolini M, Juárez ME, Cifuentes L, Hübner ME, Pardo RA. Prevalencia al nacimiento de aberraciones cromosómicas en el Hospital Clínico de la Universidad de Chile. Período 1990-2000. Rev Med Chile. 2003;131:651-58.

3. Weijerman ME, de Winter JP. Clinical practice: The care of children with Down syndrome. Eur J Pediatr. 2010;169:144552 .

4. Bittles AH, Glasson EJ. Clinical, social and ethical implications of changing life expectancy in Down syndrome. Dev Med Child Neurol. 2004;46:282-6.

5. Retamales N, Moreno R, González A, Cerda J, Lizama M. Morbilidad y Mortalidad durante el Primer Año de Vida en Pacientes con Síndrome de Down. Rev Chil Pediatr. 2009;80(4):32331.

6. Dawson $\mathrm{Al}$, Cassell $\mathrm{CH}$, Oster $\mathrm{ME}$, et al. Hospitalizations and associated costs in a population-based study of children with Down syndrome born in Florida. Birth Defects Res A Clin Mol Teratol. 2014;100:826-36.

7. Bruijn $M$, van der Aa LB, van Rijn RR, Bos AP, van Woensel JB. High incidence of acute lung injury in children with Down syndrome. Intensive Care Med. 2007;33:2179-82.

8. Garrison MM, Jeffries $\mathrm{H}$, Christakis DA. Risk of death for children with Down syndrome and sepsis. J Pediatr. 2005;147:748-52.

9. Fudge JC Jr, Li S, Jaggers J, et al. Congenital heart surgery outcomes in Down syndrome: analysis of a national clinical database. Pediatrics. 2010;126:315-22.

10. Pallardó FV, Degan P, d'Ischia M, et al. Multiple evidence for an early age prooxidant state in Down Syndrome patients. Biogerontology. 2006;7:211-20.

11. Zitnanová I, Korytár P, Aruoma OI, et al. Uric acid and allantoin levels in Down syndrome: antioxidant and oxidative stress mechanisms? Clin Chim Acta. 2004;341:139-46.

12. Garlet TR, Parisotto EB, de Medeiros Gda S, et al. Systemic oxidative stress in children and teenagers with Down syndrome. Life Sci. 2013;93(16):558-63.

13. He J, Li T, Chen J, et al. Plasma antioxidant enzymes and lipoperoxidation status in children with Down syndrome. Clin Biochem. 2016;49(1):61-5.

14. Brooksbank BW, Balazs R. Superoxide dismutase, glutathione peroxidase and lipoperoxidation in Down's syndrome fetal brain. Brain Res. 1984;318:37-44.

15. Watts R, Vyas H. An overview of respiratory problems in children with Down's syndrome. Arch Dis Child. 2013;98:812-7.

16. Lizama M, Cerda J, Monge M, et al. Morbimortalidad hospitalaria en niños con síndrome de Down. Rev Chil Ped. 2016;87:102-9.

17. Byard RW. Forensic issues in Down syndrome fatalities. J Forensic Leg Med. 2007;14:475-81.

18. Amaral Loureiro AC, Oliveira Costa F, Eustáquio da Costa J. The impact of periodontal disease on the quality of life of individuals with Down syndrome. Downs Syndr Res Pract. 2007;12:50-4.

19. Mitchell RB, Call E, Kelly J. Ear, nose and throat disorders in children with Down syndrome. Laryngoscope. 2003;113:25963.

20. De Hingh YCM, Van der Vossen PW, Gemen EF, et al. Intrinsic abnormalities of lymphocyte counts in children with Down syndrome. J Pediatr. 2005;147:7447.

21. Bertrand P, Navarro H, Caussade S, Holmgren N, Sánchez I. Airway anomalies in children with Down syndrome: endoscopic findings. Ped Pulmonol. 2003;36:137-41.

22. Hamilton J, Yaneza MM, Clement WA, Kubba $\mathrm{H}$. The prevalence of airway problems in children with Down's syndrome. J Int J Pediatr Otorhinlaryngol. 2016; 81:1-4

23. McDowell KM, Craven DI. Pulmonary complications of Down syndrome during childhood. J Pediatrics. 2011;158(2):31925.

24. Taytard J, Nathan N, de Blic J, et al. New insights into pediatric idiopathic pulmonary hemosiderosis: the French RespiRare $^{\circledR}$ cohort. Orphanet J Rare Dis. 2013;8:161.

25. Zachariah P, Ruttenber M, Simôes EA. Down syndrome and hospitalizations due to Respiratory Syncytial Virus: a population-based study. J Pediatr. 2012;160:827-31.

26. Lee YI, Peng CC, Chiu NC, Huang DT, Huang FY, Chi H. Risk factors associated with death in patients with severe respiratory syncytial virus infection. J Microbiol Immunol Infect. 2016;49(5):737-42.

27. Shott SR. Down Syndrome: Analysis of Airway Size and a Guide for Appropriate Intubation. Laryngoscope. 2000;110(4):585-92.

28. Watts R, Vyas H. An overview of respiratory problems in children with Down's syndrome. Arch Dis Child. 2013;98:812-7.

29. Galambos C. Increased Lung Expression of Anti-Angiogenic Factors in Down
Syndrome: Potential Role in Abnormal Lung Vascular Growth and the Risk for Pulmonary Hypertension. PLoS One. 2016 3;11(8):e0159005.

30. Saji T. Clinical characteristics of pulmonary arterial hypertension associated with Down syndrome. Pediatr Int. 2014;56(3):297-303.

31. Gowda S, Bhat D, Feng Z, Chang CH, Ross RD. Pulmonary vein stenosis with Down syndrome: a rare and frecuently fatal cause of pulmonary hypertension in infants and children. Congenit Heart Dis. 2014;9:e90-7.

32. Tibby SM, Durward A, Goh CT, et al. Clinical course and outcome for critically ill children with Down syndrome: a retrospective cohort study. Intensive Care Med. 2012;38:1365-71.

33. Valle P, Ronco R, Clavería C, et al. Extubación fallida en pacientes pediátricos después de cirugía de cardiopatías congénitas. Rev Chil Pediatr. 2005;76(5):479-84.

34. Freeman SB, Bean LH, Allen EG, et al. Ethnicity, sex, and the incidence of congenital heart defects: a report from the National Down Syndrome Project. Genet Med 2008; 10:173-80.

35. Irving CA, Chaudhari MP. Cardiovascular abnormalities in Down's syndrome: spectrum, management and survival over 22 years. Arch Dis Child. 2012;97:326-30.

36. Tóth R, Szántó P, Prodán Z, et al. Down syndrome and postoperative complications after paediatric cardiac surgery: a propensity-matched analysis. Interact Cardiovasc Thorac Surg. 2013;17:691-7.

37. Tumanyan MR, Filaretova OV, Chechneva VV, Gulasaryan RS, Butrim IV, Bockeria LA. Repair of complete atrioventricular septal defect in infants with down syndrome: outcomes and long-term results. Pediatr Cardiol. 2015;36(1):71-5.

38. Kraemer FW, Stricker PA, Gurnaney HG, et al. Bradycardia during induction of anesthesia with sevoflurane in children with Down syndrome. Anesth Analg. 2010; 111(5):1259-63.

39. Bai W, Voepel-Lewis T, Malviya S. Hemodynamic changes in children with Down syndrome during and following inhalation induction of anesthesia with sevoflurane. J Clin Anesth. 2010;22:592-7.

40. Kovacikova L, Skrak P, Dobos D, Zahorec $\mathrm{M}$. Noninvasive positive pressure ventilation in critically ill children with cardiac disease. Pediatr Cardiol. 2014;35:676-83.

41. Evans JM, Dharmar M, Meierhenry E, Marcin JP, Raff GW. Association between Down syndrome and in-hospital death among children undergoing surgery for congenital heart disease: a US population- 
based study. Circ Cardiovasc Qual Outcomes 2014;7:445-52.

42. Ram G, Chinen J. Infections and immunodeficiency in Down syndrome. Clin Exp Immunol. 2011;164(1):9-16.

43. Cvijanovich N, Shanley TP, Lin R, et al. Validating the genomic signature of pediatric septic shock. Physiol Genomics. 2008;34:127-34.

44. Cvijanovich N, King JC, Flori HR, Gildengorin G, Wong HR. Zinc homeostasis in pediatric critical illness. Pediatr Crit Care Med. 2009;10:29-34.

45. Wong HR, Shanley TP, Sakthivel B, et al. Genome-level expression profiles in pediatric septic shock indicate a role for altered zinc homeostasis in poor outcome. Physiol Genomics. 2007;30:146-55.

46. Lima AS, Cardoso BR, Cozzolino SF. Nutritional status of Zinc in children with Down syndrome. Biol Trace Elem Res. 2010;133:20-8

47. Schieve LA, Boulet SL, Boyle C, Rasmussen SA, Schendel D. Health of children 3 to 17 years of age with Down syndrome in the 1997-2005 national health interview survey. Pediatrics. 2009; 123 (2): e253-60.

48. Gaete B, Mellado C, Hernández M. Prevalence of neurological disorders among children with Down syndrome. Rev Med Chile. 2012;140(2):214-8.

49. Smigielska-Kuzia J, Sobaniec W, Kulak W, Bockowski L. Clinical and EEG features of epilepsy in children and adolescents in Down syndrome. J Child Neurol 2009;24(4):416-20.

50. Arya R, Kabra M, Gulati S. Epilepsy in children with Down syndrome. Epileptic Disord. 2011;13(1):1-7.

51. Verrotti A, Cusmai R, Nicita F, et al. Electroclinical features and long-term outcome of cryptogenic epilepsy in children with Down syndrome. J Pediatr. 2013;163:1754-8.

52. Sanmaneechai O, Sogawa Y, Silver W, Ballaban-Gil K, Moshé SL, Shinnar S. Treatment outcomes of West syndrome in infants with Down syndrome. Pediatr Neurol. 2013;48:42-7.

53. Sobey CG, Judkins CP, Sundararajan V, Phan TG, Drummond GR, Srikanth VK. Risk of Major Cardiovascular Events in People with Down Syndrome. PLoS One. 2015;10(9):e0137093.

54. Dai AI, Shaikh ZA, Cohen ME. Earlyonset Moyamoya syndrome in a patient with Down syndrome: Case report and review of the literature. J Child Neurol. 2000;15:696-9.

55. Jea A, Smith ER, Robertson R, Scott RM. Moyamoya syndrome associated with Down syndrome: outcome after surgical revascularization. Pediatrics. 2005;116:e694-e701.

56. Tassone JC, Duey-Holtz A. Spine concerns in the special Olympian with Down syndrome. Sports Med Arthrosc. 2008; 16:55-60.

57. Birrer RB. The special olympics athlete: evaluation and clearance for participation. Clin Pediatr. 2004;43:777-82.

58. Lewanda AF, Matisoff A, Revenis M, et al. Preoperative evaluation and comprehensive risk assessment for children with Down syndrome. Paediatr
Anaesth. 2016;26(4):356-62.

59. Borland LM, Colligan J, Brandom BW. Frequency of anesthesia-related complications in children with Down syndrome under general anesthesia for noncardiac procedures. Pediatr Anesth. 2004;14:733-8.

60. Hata T, Todd MM. Cervical spine considerations when anesthetizing patients with Down syndrome. Anesthesiology. 2005;102:680-5.

61. Van Driest SL, Shah A, Marshall MD, et al. Opioid use after cardiac surgery in children with Down syndrome. Pediatr Crit Care Med. 2013;14(9):862-8.

62. Terada Y, Tachibana K, Takeuchi M, Kinouchi K. Comparison of Sedative and Analgesic Requirements in Children with and without Down Syndrome following Pediatric Cardiac Surgery. Masui. 2016;65(1):56-61.

63. Valkenburg AJ, Calvier EA, van Dijk $\mathrm{M}$, et al. Pharmacodynamics and Pharmacokinetics of Morphine After Cardiac Surgery in Children With and Without Down Syndrome. Pediatr Crit Care Med. 2016;17(10):930-8.

64. Gupta P, Rettiganti M. Association between Down syndrome and mortality in young children with critical illness: a propensity-matched analysis. Acta Paediatr. 2015;104(11):e506-11.

65. Joffre C, Lesage F, Bustarret O, Hubert P, Oualha M. Children with Down syndrome: Clinical course and mortalityassociated factors in a French medical paediatric intensive care unit. J Paediatr Child Health. 2016;52(6):595-9. 